\title{
Transcatheter aortic valve replacement using the transaortic approach
}

\author{
Michael Caskey ${ }^{1}$, Hao Pan ${ }^{2}$, Merrick Kirshner ${ }^{1}$, Timothy Byrne ${ }^{1}$, Divya Ratan Verma ${ }^{3}$ James Flaherty ${ }^{4}$, \\ Andrei Churyla ${ }^{4}$ \\ ${ }^{1}$ Arizona Heart Hospital, Phoenix, AZ, USA; ${ }^{2}$ University of Texas Health Science Center at San Antonio, San Antonio, TX, USA; Banner University \\ Medical Center, Phoenix, AZ, USA; ${ }^{4}$ Northwestern Memorial Hospital, Chicago, IL, USA \\ Correspondence to: Michael Caskey, MD. Southwest Heart and Lung, 10930 North Tatum Blvd. Ste. 103, Phoenix, AZ 85028, USA. \\ Email: micaskey@icloud.com.
}

Submitted Jul 31, 2017. Accepted for publication Aug 28, 2017.

doi: 10.21037/acs.2017.09.05

View this article at: http://dx.doi.org/10.21037/acs.2017.09.05

\section{Clinical vignette}

\section{Clinical history}

This is a frail 73-year-old male with symptomatic critical aortic stenosis (AS). He has suffered a significant functional decline with associated shortness of breath and chest discomfort. His past medical history is significant for coronary artery disease with prior bypass grafting, porcelain aorta, chronic kidney disease-stage III, diabetes mellitus, hypertension, hyperlipidemia, and peripheral arterial disease.

Echocardiography demonstrates tri-leaflet, calcific AS with an aortic valve area of $0.7 \mathrm{~cm}^{2}$, a jet velocity of $5.0 \mathrm{~m} / \mathrm{s}$, and a mean gradient of $60 \mathrm{mmHg}$. Cardiac catheterization reveals a widely patent LIMA-to-LAD with patent vein grafts and limited native coronary flow.

\section{Heart team considerations}

The patient clearly has symptomatic, critical AS and meets high-risk/inoperable criteria. Based on this, the Heart Team recommended transcatheter aortic valve replacement (TAVR). However, after reviewing the CT angiogram, two things became clear. First, with left ventricular outflow tract calcification and a large annular size $(90 \mathrm{~mm}$ annular perimeter), it was decided that a self-expanding valve would be the best choice for this case; thus, a $34 \mathrm{~mm}$ Evolut R (Medtronic, Inc., Minneapolis, MN, USA) transcatheter aortic valve was selected. Second, standard transfemoral access could not be performed because of extensive iliofemoral disease. There was also extensive arteriosclerosis of the aortic arch and ascending aorta with a patent LIMAto-LAD, which prevented the use of a subclavian or carotid approach. Therefore, the Heart Team felt the Transaortic (TAo) or direct aortic (DA) approach would be the best way to implant the prosthesis.

\section{Surgical plan}

There are two ways to approach a TAo TAVR. The first is a mini-J sternotomy, which gives full access to the upper ascending aortic. The second is a right parasternal approach. Typically, the right parasternal approach has more limited aortic access and is usually reserved for rather steep aortic angulation, with at least $50 \%$ of the aortic diameter to the right of the right sternal margin on CT.

Based on the CT findings, we decided to use the right parasternal approach. This would require resection of the head of the third rib and placing purse strings on the high right lateral aortic wall approximately $8 \mathrm{~cm}$ distal to the aortic annulus. Pre-implantation balloon aortic valvuloplasty (BAV) would be not performed, and implantation of the prosthesis was planned to be at the standard depth of 3 to $5 \mathrm{~mm}$. We also planned to avoid post-implantation BAV, if possible, because of heavy sino-tubular junction (STJ) calcification.

\section{Surgical techniques}

\section{Preparation}

We use a hybrid room for all TAVR cases. 
Our multi-disciplinary TAVR team consists of a cardiac surgeon, a cardiac anesthesiologist with intraoperative transesophageal echocardiography (TEE) experience, an interventional cardiologist, a perfusionist, a surgical scrub nurse cross-trained in transcatheter procedures, a radiology technician, and a circulating nurse trained in valve preparation and loading.

Standard patient positioning is used. The C-arm gantry must be positioned on the patient's left side or come in from the top. The display monitors must be positioned so that both operators have access to images and hemodynamics. We do standard open heart prep for all patients; general anesthesia is mandatory and transesophageal echocardiography (TEE) is preferred. The anesthesiologist places a central venous line, preferably into the right internal jugular vein, and a radial arterial line for continuous hemodynamic monitoring.

\section{Exposition}

Typically, the surgeon stands to the patient's right side and begins the procedure while the interventional cardiologist simultaneously obtains femoral arterial and venous access. A $6 \mathrm{Fr}$ femoral arterial sheath is placed so that a pigtail catheter can be inserted to obtain an aortic root arteriogram; if needed, the femoral arterial sheath can also be used for rescue cardiopulmonary bypass (CPB). A $5 \mathrm{Fr}$ femoral venous sheath is placed for insertion of a temporary transvenous pacemaker lead; if necessary the femoral venous sheath can be used to provide venous access for rescue $\mathrm{CPB}$. Both of the equipment tables, the cath table, and the surgical equipment trays are immediately behind the operating team. Once surgical access has been established, the cath lab table is pulled up to the patient.

\section{Operation}

\section{Incision}

As noted earlier, the right parasternal approach was used for this case. Making the incision is often one of the more critical steps in the case. We place a marker pigtail catheter from the groin into the aortic root. We then count up $8 \mathrm{~cm}$ from the annulus and lay a corresponding surgical clamp on the chest wall. This becomes our incision site, as seen in the video.

A 3- to 4-inch transverse incision is made to the right edge of the sternum, typically over the third rib. The exposed rib is identified and resected. This usually requires ligation of the right internal mammary artery and vein.

A hand port/soft tissue retractor is placed, broadening exposure. Dissection is then carried down to the pericardium and stay sutures are placed, exposing the aorta.

Epi-aortic ultrasound can be useful, but is not required. Two standard pledgeted purse string sutures are placed using good tissue bites.

\section{Cannulation}

One of two methods can be used to place the large delivery sheath: a counter incision-type approach, which lowers the angle of incidence into the aorta, or direct aortic cannulation through the wound. With either method, a needle is inserted through the skin or through the wound directly into the aorta. Then, a wire, small sheath, and catheter are passed through the aorta to the root to facilitate crossing the valve. After crossing, typically with an AL-1 guide catheter, exchanges are completed, trading up to a pigtail. Left ventricular and aortic valve hemodynamics are obtained. We then place a stiff wire with a loop at its tip in the left ventricular apex. Our preference is a Confida (Medtronic, Inc., Minneapolis, Minnesota) or a Lunderquist (Cook Medical, Bloomington, Indiana) guidewire. A $20 \mathrm{Fr}$ sheath, such as a Gore DrySeal (W. L. Gore \& Associates, Inc., Newark, Delaware) or a Cook, can now be passed over the stiff left ventricular wire and positioned $2 \mathrm{~cm}$ deep into the aorta. Typically, a bumper is placed $2 \mathrm{~cm}$ up the sheath to prevent deeper migration. The sheath must be doubly secured and firmly held.

\section{Deployment}

At this point, a pre-BAV can be completed if necessary. The delivery catheter is now passed over the wire into the sheath to the desired delivery depth $(3-5 \mathrm{~mm})$ while simultaneously maintaining the proper sheath depth. The proper co-planer view is established, and then parallax of the bottom of the valve is obtained, usually by moving the $\mathrm{C}$-arm gantry caudal. Slow delivery is initiated, utilizing controlled pacing at $90 \mathrm{bpm}$, until full annular contact is made. This is followed by deployment of the valve to $80 \%$. Note that with an Evolut R implant, it is typical to have asymmetric frame positioning at $80 \%$ deployment. The more common position is a deeper frame depth on the left coronary side and less so on the non-coronary cusp (NCC) side. The frame usually auto corrects, but only with caveats.

We prefer to always start with a relatively shallow $(2-3 \mathrm{~mm}) \mathrm{NCC}$ depth. If there is at least moderate calcification in the annulus/root, forward tension on the 
delivery catheter during a very slow deployment will facilitate the proper auto-correction. With lesser amounts of calcification, forward tension on the delivery catheter can result in an auto-corrected frame that is too deep. So, in an aortic root with minimal calcification, the delivery catheter should be held in more of a neutral position initially while the frame is very slowly deployed, giving back only a slight forward tension at the final stages of release.

The valve is then assessed for depth and perivalvular leak (PVL) by aortogram and TEE. In this case, the valve was recaptured and repositioned to a slightly shallower depth. At this point, the valve is fully deployed and assessed.

As seen on the video, there was moderate PVL and a mean gradient of $17 \mathrm{mmHg}$. Because of a narrow STJ $(24 \mathrm{~mm})$ with extensive calcification, a $23 \mathrm{~mm} \mathrm{Z}$-Med balloon was used for post-BAV. This reduced the mean gradient to $7 \mathrm{mmHg}$ and PVL to mild. This concluded the deployment phase.

\section{Completion}

After all valve-related interventions are completed, the stiff left ventricle wire is removed utilizing a pigtail catheter. The sheath is then removed. Both purse strings are tied down and an additional stitch may be placed if necessary. The pericardium is usually left open. A small, $24 \mathrm{~mm}$ Silastic drain is placed to drain the space. The lung is then reinflated, and the chest wall closed securely in layers. Marcaine $0.25 \%$ is then injected locally.

\section{Comments}

\section{Clinical results}

TAo approach TAVR has been shown to be safe and feasible (1). Whether performed through a mini-sternotomy or a right parasternal approach, TAo TAVR is a valuable tool to have in your toolbox. Initially, alternative access was useful in just over $15 \%$ of all TAVR cases (1). Currently, with decreasing catheter size and increasing experience, the transfemoral approach is used $96 \%$ of the time (1). The learning curves for trans-apical and transaortic approaches are distinct, but technical proficiency begins to develop by 25 cases and becomes complete by 50 cases for both approaches (1). Given the relatively low volume of alternative access cases, achieving technical proficiency may take significant time. However, recent studies have shown that technical proficiency had no effect on 30-day or 1-year mortality for any access approach (1).

Based on our experience, we have not performed a TAo approach case in at least 18 months. Our current strategic approach when using the Evolut R TAVR system is: transfemoral, left subclavian, right carotid, then TAo, if necessary. Other approaches are currently under investigation. In our program, right subclavian, trans-apical and transcaval alternate approaches are rarely used.

\section{Advantages}

The TAo approach provides a feasible alternative for patients with challenging anatomic features that may otherwise preclude use of the TAVI procedure (2). Advantages of this approach include a more controlled approach and a precise depth of implant. With the deployment site being no more than 6 to 12 inches from the entrance of the sheath, a precise depth of implant is typically readily achieved. However, even with these advantages comes the disadvantage of having to do a nonpercutaneous approach. Despite this, the TAo approach is surprisingly well-tolerated in the majority patients.

\section{Caveats}

TAo approach TAVR is a great foundational tool for those cases where all other approaches are not suitable. However, this approach is not without its challenges. Current usage has become infrequent and, therefore, many lessons may need to be relearned.

Sheath migration during catheter manipulation and deployment remains the Achilles' heel of this procedure. Placing a small, secure bumper $2 \mathrm{~cm}$ up from the end of the sheath tends to obviate this problem. There have also been reported problems with bleeding, aortic dissection, and recurrent right pleural effusions. Also, it is important to avoid a parasternal approach for bicuspid disease with a thin ascending aorta. The safety margin can be quite narrow (3). Despite this, TAo approach TAVR has been shown to be safe and feasible.

\section{Acknowledgements}

None.

\section{Footnote}

Conflicts of Interest: The authors have no conflicts of interest 
to declare.

\section{References}

1. Henn MC, Percival T, Zajarias A, et al. Learning alternative access approaches for transcatheter aortic valve replacement: Implications for new transcatheter aortic valve replacement centers. Ann Thorac Surg

Cite this article as: Caskey M, Pan H, Kirshner M, Byrne T, Verma DR, Flaherty J, Churyla A. Transcatheter aortic valve replacement using the transaortic approach. Ann Cardiothorac Surg 2017;6(5):561-564. doi: 10.21037/acs.2017.09.05
2017;103:1399-405.

2. Bruschi G, Branny $M$, Schiltgen $M$, et al. One-year outcomes of transcatheter aortic valve implantation using the direct aortic approach. Ann Thorac Surg 2017;103:1434-40.

3. Chow SCY, Cheung GSH, Lee APW, et al. Transcatheter aortic valve implantation: the transaortic approach. Asian Cardiovasc Thorac Ann 2017:25:357-63. 